\title{
Imported cutaneous leishmaniasis caused by Leishmania major in a Chinese laborer who worked in Saudi Arabia*
}

\author{
Min Zhang ${ }^{1}$ \\ Haibo Liu ${ }^{2}$ \\ Hong Sang ${ }^{1}$
}

\author{
Fang Liu ${ }^{1}$ \\ Wenxing $\mathrm{Hu}^{2}$
}

DOI: http:/ / dx.doi.org/10.1590/abd1806-4841.20163820

\begin{abstract}
We report an imported case of cutaneous leishmaniasis in a 37-year-old man from Saudi Arabia caused by Leishmania major. He presented with non-healing nodulo-ulcerative lesions with a "volcanic crater" on the lower limbs. It was clearly cutaneous leishmaniasis - a rare disease in China - as reflected by the patient's clinical history, the lesions' morphology, histopathological examination, culture and PCR analysis of the lesions. The patient was completely cured after two cycles of sodium stibogluconate treatment. This case report demonstrates that dermatologists should be aware of sporadic cutaneous leishmaniasis cases in non-endemic areas.
\end{abstract}

Keywords: Leishmania major, Leishmaniasis, cutaneous, Parasites

\section{INTRODUTION}

Leishmaniasis, a major world health problem, is increasing in incidence because of tourism and job-related travel ${ }^{1}$. Depending on the Leishmania species and the host's immune status, different forms may develop, including cutaneous leishmaniasis (CL), mucocutaneousleishmaniasis (MCL) or visceral leishmaniasis (VL). About $90 \%$ of CL is endemic in the tropics and neotropics, in countries such as Afghanistan, Algeria, Brazil, Iran, Peru, Saudi Arabia and Syria ${ }^{2}$. Here we report a case of $C L$ acquired by a Chinese laborer who worked in Saudi Arabia.

\section{CASE REPORT}

A 37-year-old, previously healthy man presented to our department with a non-healing nodulo-ulcerative lesion for 3 months. The lesion started as a red papule and developed into asymptomatic swelling within a few weeks and gradually extended. Subsequently, the lesions became multiple, raised, well-defined, ulcerated, developing into crusted nodules of variable size on the lower limbs (Figure 1A). He worked on railroads in Saudi Arabia for one year, beginning in September 2011, including night shifts. Dermatological examination revealed multiple nodules in a zosteriform distribution and several ulcerative or nodulo-ulcerative lesions with a "volcanic crater" on the lower limbs (Figure 1B). When he was hospitalized, biopsies were taken. Histopathologic examination showed amastigotes in histiocytes (Figure 2A and 2B). Leishmaniasis was suspected, clinical samples for the smears, and tissue were sent for polymerase chain reaction (PCR) analysis and culture.

Smears were fixed in methanol, Giemsa-stained, and microscopically examined for the presence of amastigotes. Cultures were set up in the Novy-McNeal-Nicolle (NNN) medium, and microscopically examined for promastigotes every 2 days. On day 10, promastigotes were observed (Figure 2C). Further sequencing of the ITS-1 and ITS-2 PCR product showed $100 \%$ homology with standard L. major (FM209179.1). 
The patient was diagnosed with CL based on the patient's clinical history, thelesions' morphology, stained smear test, histopathological examination, culture and PCR analysis.
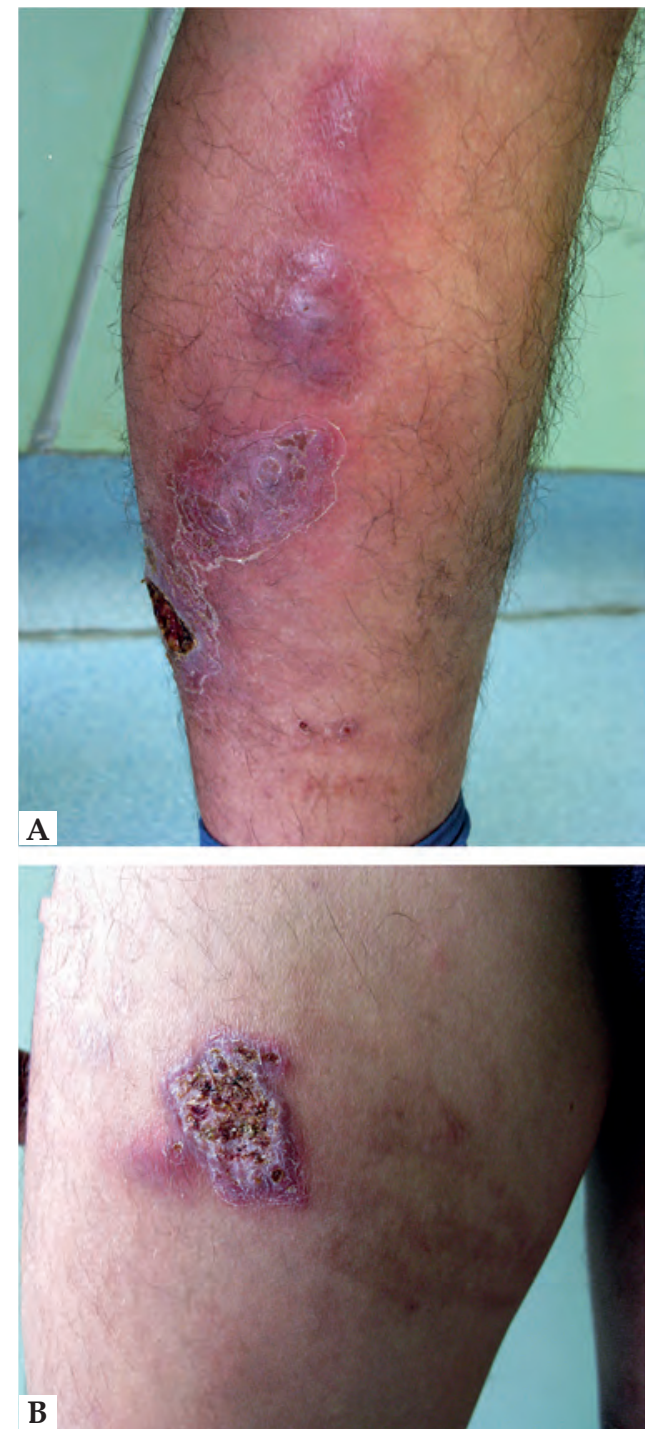

Figure 1 :

A: $\quad$ Multiple nodules in a zosteriform distribution and an ulcer on the left leg B: An ulcer with infiltrated border and a satellite nodule on the right leg
He was treated with intramuscular injections of sodium stibogluconate $600 \mathrm{mg} / \mathrm{d}$ for 10 days; the same treatment was repeated once more.As the lesions had significantly improved, the patient was discharged (Figure 3A and 3B). Post-treatment evaluation after 6 weeks, including direct microscopy, culture and
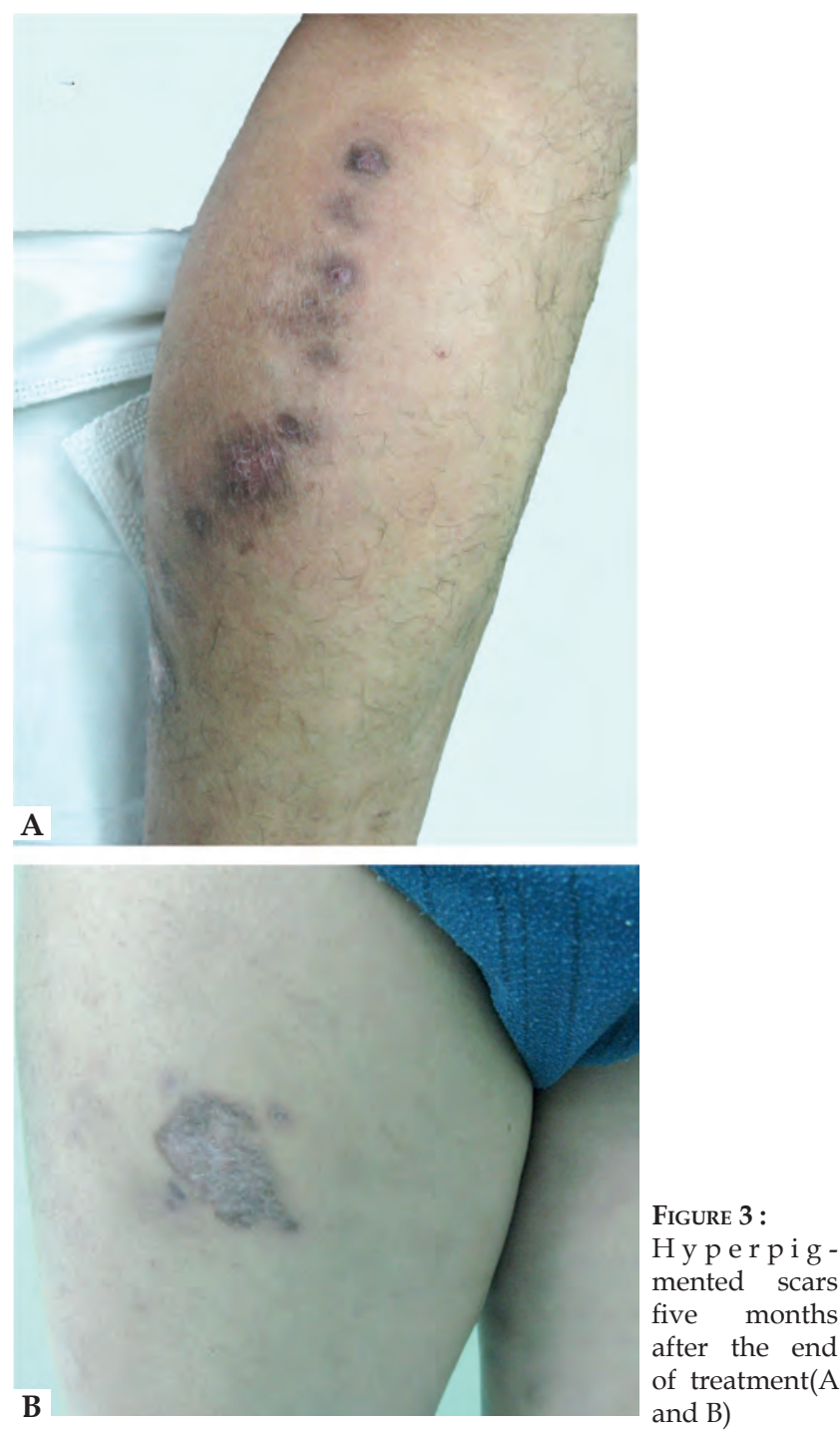
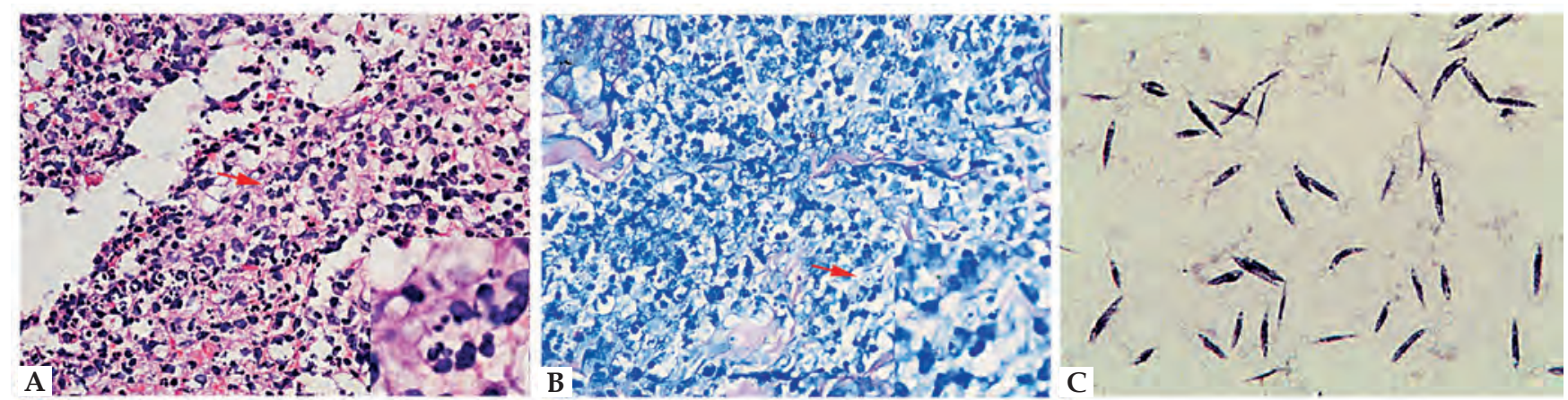

FIGURE 2 : Histology of biopsy revealed amastigotes in histiocytes (A HE×400, B Wright stain $\times 400$ ), (C) promastigotes were observed in NNN-medium (Giemsa×1000). 
PCR revealed no evidence of residual disease. The patient was followed up for 2 years with no recurrence.

\section{DISCUSSION}

Cutaneous Leishmaniasis was first described by Moursy in Saudi Arabia in 1976. ${ }^{3}$ It is recognized as a complex and highly variable disease in terms of its epidemiology, etiology, pathology and clinical presentation. It can be caused by several Leishmaniaspp, entailing the major species of L. tropica and L. major in the Old World, and L. infantum, L. amazonensis, L. mexicana and L.venezuelensis in the New World. ${ }^{1}$ In endemic countries, it occurs more often in children than in adults. The most frequently affected sites are the face and extremities. CL typically presents as papules, crusted nodules, plaques, or nodulo-ulcerative lesions. ${ }^{4} \mathrm{CL}$ lesions may simulate other conditions such as tumor-like lesions, when ulcerated, they may resemble basal cell carcinomas, especially in older individuals, making diagnosis difficult. To maximize sensitivity and specificity of diagnosis, examinations including direct microscopy, histopathology, culture and PCR should ideally be performed.

In China, CL induced by L. infantum only occurred in Karamay, Xin jiang, with a prevalence of 1.0$1.6 \%$. Our patient represented a rare case of import- ed CL induced by L. major whilst working in Saudi Arabia. According to the literature, CL caused by $L$. major is a growing, endemic, public health problem in many parts of the Kingdom of Saudi Arabia. ${ }^{4}$ Most of patients reported were non-Saudi expatriate workers who suffered mostly from multiple and severely inflamed nodulo-ulcerative lesions on exposed body parts. The vector is Phlebotomuspapatasi and desert rodents are the main animal reservoirs. But CL can represent a diagnostic challenge in non-endemic areas where experience is limited.

Treatment aims to accelerate healing, reduce the risk of scarring and prevent disease progression. The choice of treatment depends on lesion size and location, the number of lesions and the potential for dissemination. Pentavalent, antimonial drugs (sodium stibogluconate or meglumineantimonate) are the traditionally accepted first-line agents. Miltefosine ${ }^{5}$ has recently emerged as an agent to treat leishmaniasis, offering significant advantages like good oral bioavailability and tolerability. Using intravenous liposomal amphotericin B, Harefuah successfully treated $\mathrm{CL}$ in a 1-year-old boy with multiple skin lesions, free of complications. ${ }^{6}$ Other successful therapies such as azithromycin, fluconazole and itraconazole have also been reported. $\left.{ }^{7-9}\right]$

\section{REFERENCES}

1. Ameen M. Cutaneous and mucocutaneousleishmaniasis: emerging therapies and progress in disease management. Expert OpinPharmacother. 2010;11:557-69.

2. Ameen M. Cutaneous leishmaniasis: advances in disease pathogenesis, diagnostics and therapeutics. Clin ExpDermatol. 2010;35:699-705.

3. Morsy TA, Shoura MI.Some aspects of cutaneous leishmaniasis in Riyadh. J Trop Med Hyg. 1976;79:137-9

4. Al-Tawfiq JA, AbuKhamsin A. Cutaneous leishmaniasis: a 46-year study of the epidemiology and clinical features in Saudi Arabia (1956-2002). Int J Infect Dis. 2004;8:244-50.

5. Dorlo TP, van Thiel PP, Schoone GJ, Stienstra Y, van Vugt M, Beijnen JH, et al. Dynamics of parasite clearance in cutaneous leishmaniasis patients treated with miltefosine. PloSNeglTrop Dis. 2011;5:e1436.

6. Ben-Shimol S, Sagi O, Schwartz E, Greenberg D. [Cutaneous leishmaniasis treated with ambisome (liposomalamphotericin B)]. Harefuah. 2012;151:458-60, 498.

7. White JM, Salisbury JR, Jones J, Higgins EM, Vega-Lopez F. Cutaneous leishmaniasis: three children with Leishmania major successfully treated with itraconazole. Pediatr Dermatol. 2006;23:78-80.

8. Sklavos AV, Walls T, Webber MT, Watson AB.Cutaneous leishmaniasis in a child treated with oral fluconazole. Australas J Dermatol. 2010;51:195-7.

9. Minodier P, Zambelli L, Mary C, Faraut F, Garnier JM, Berbis P. Cutaneous leishmaniasis treated with azithromycin in a child. Pediatr Infect Dis J. 2008;27:80-1.

\author{
MAILING ADDRESS: \\ Hong Sang \\ Department of Dermatology \\ Jinling Hospital affiliated to Nanjing University \\ 210002 Nanjing, China. \\ E-mail: sanghong@nju.edu.cn
}

How to cite this article: Zhang M, Liu F, Liu HB, Hu WX, Sang H. Imported cutaneous leishmaniasis caused by Leishmania major in a Chinese labour who worked in Saudi Arabia. An Bras Dermatol. 2016;91(3):365-7. 